\title{
Recommendations on screening for lung cancer
}

\author{
Canadian Task Force on Preventive Health Care*
}

CMAJ Podcasts: author interview at https://soundcloud.com/cmajpodcasts/151421-guide

$\mathrm{L}$ ung cancer is the most common cause of cancer-related deaths and the most commonly diagnosed cancer among Canadians - an estimated 26600 Canadians were diagnosed and 20900 died from lung cancer in 2015. ${ }^{1}$ In Canada, the incidence of lung cancer is currently higher in men than in women (although this gap is beginning to narrow), and more than $85 \%$ of cases are related to smoking tobacco. About $44 \%$ of Canadians (12.6 million) smoke or have quit smoking. ${ }^{2}$ Those with a history of heavy smoking are at the greatest risk for lung cancer. Smoking history is often measured in pack-years, which is the product of the average number of packs smoked daily and the number of years of smoking (e.g., individuals who smoked one pack a day [20 cigarettes] for 30 years, or two packs a day for 15 years, would both have a 30 pack-year history).

Mortality is extremely high in late-stage lung cancer but much lower in earlier stages (5-yr relative survival rates for stage 4 and $1 \mathrm{~A}$ lung cancer were $1 \%$ and $49 \%$, respectively, in 2007). ${ }^{3}$ Screening for lung cancer aims to detect disease at an early stage, when it may respond better to treatment and be less likely to cause serious illness or death. Therefore, such screening has been proposed as an adjunct to other methods for reducing the burden of lung cancer, including global tobacco control initiatives and interventions designed to encourage smoking cessation. ${ }^{4}$ No organized lung cancer screening programs have been implemented in Canada.

\section{Scope}

This guideline is intended to provide primary care providers and policy-makers with guidance on screening for lung cancer, and replaces the previous 2003 Canadian Task Force on Preventive Health Care recommendations. ${ }^{5}$ Since the previous recommendations were published, results from additional studies have been released, including one large randomized controlled trial (RCT) comparing lowdose computed tomography (CT), which uses less ionizing radiation than usual CT scans, with chest radiography. ${ }^{6}$ Ongoing trials of screening with lowdose $\mathrm{CT}^{7-10}$ are expected to provide more evidence on the effectiveness of screening for lung cancer with low-dose CT. The current recommendations may be updated once these results are available or at least within five years.

\section{Methods}

The Canadian Task Force on Preventive Health Care is an independent panel of clinicians and methodologists that makes recommendations about clinical manoeuvres aimed at primary and secondary prevention (www.canadiantaskforce. ca). These recommendations were developed by a work group of six members of the task force and scientific staff at the Public Health Agency of Canada. ${ }^{11}$
Competing interests: None declared.

This article has been peer reviewed.

*The authors of this article are listed at the end of the article, under "guideline writing group.'

The complete list of current members of the Canadian Task Force on Preventive Health Care is available at www.canadiantaskforce.ca/ members_eng.html

Correspondence to: Canadian Task Force on Preventive Health Care, info@canadiantaskforce.ca

CMAJ 2016. DOI:10.1503 /cmaj.151421

\section{KEY POINTS}

- Adults between 55 and 74 years of age who are at high risk for lung cancer (i.e., those who smoke or who quit smoking within the past 15 years and who have at least a 30 pack-year smoking history) may benefit from screening for lung cancer with low-dose computed tomography (CT) every year for three consecutive years (weak recommendation).

- This weak recommendation implies that practitioners should have a discussion with their patients about the benefits and harms of screening for lung cancer with low-dose CT that includes information about false-positive results, adverse effects of invasive follow-up testing and overdiagnosis.

- Because of the potential for screening-related harms, low-dose CT and subsequent management should be done in health care settings with expertise in early diagnosis and treatment of lung cancer.

- Over 6.5 years, 322 people would need to be screened with low-dose $\mathrm{CT}$ to prevent one death from lung cancer.

- Because smoking is associated with $85 \%$ of incident lung cancer cases in Canada, tobacco control and smoking cessation are critical for reducing the morbidity and mortality owing to lung cancer.

- There is no clear benefit of low-dose CT screening for lung cancer in adults younger than 55 years and older than 74 years, or in those at a lower risk based on smoking history (i.e., adults who smoke with less than a 30 pack-year smoking history or adults who quit smoking more than 15 years prior).

- There is no benefit of screening for lung cancer with chest radiography (with or without sputum cytology), but there are known harms (e.g., false-positive results, adverse effects of invasive follow-up testing and overdiagnosis). 
A systematic review to inform this guideline was conducted by the Evidence Review and Synthesis Centre at McMaster University (Hamilton, Ontario), with the aid of a clinical expert, who served as an independent advisor, and scientific staff at the Public Health Agency of Canada. ${ }^{12}$ The systematic review evaluated the benefits and harms of screening for lung cancer on the outcomes of all-cause mortality, lung cancer-specific mortality, stage of lung cancer at diagnosis, rate of smoking cessation, false-positive results, adverse effects of invasive follow-up testing and overdiagnosis. ${ }^{12}$ An outline of the analytic framework with key and contextual questions is available in Appendix 1 (available at www.cmaj.ca/lookup/suppl/ doi:10.1503/cmaj.151421/-/DC1). The Evidence Review and Synthesis Centre and the task force used the Grading of Recommendations Assessment, Development and Evaluation (GRADE) system to determine the quality of evidence and strength of recommendations (see Box 1). ${ }^{13}$

The literature search was based on the search done for the 2013 Cochrane review on screening for lung cancer. ${ }^{14}$ The Cochrane review searched CENTRAL, MEDLINE, PREMEDLINE, and

\section{Box 1: Grading of recommendations}

Recommendations are graded according to the Grading of Recommendations Assessment, Development and Evaluation system (GRADE). ${ }^{13}$ GRADE offers two strengths of recommendation: strong and weak. The strength of recommendations is based on the quality of supporting evidence, degree of uncertainty about the balance between desirable and undesirable effects, degree of uncertainty or variability in values and preferences and degree of uncertainty about whether the intervention represents a wise use of resources.

Strong recommendations are those for which the Canadian Task Force on Preventive Health Care is confident that the desirable effects of an intervention outweigh its undesirable effects (strong recommendation for an intervention) or that the undesirable effects of an intervention outweigh its desirable effects (strong recommendation against an intervention). A strong recommendation implies that most individuals will be best served by the recommended course of action.

Weak recommendations are those for which the desirable effects probably outweigh the undesirable effects (weak recommendation for an intervention) or undesirable effects probably outweigh the desirable effects (weak recommendation against an intervention), but appreciable uncertainty exists. A weak recommendation implies that most people would want the recommended course of action but that many would not.

Clinicians must recognize that different choices will be appropriate for each individual, and they must help each person arrive at a management decision that is consistent with his or her values and preferences. Policy-making will require substantial debate and involvement of various stakeholders. Weak recommendations result when the balance between desirable and undesirable effects is small, the quality of evidence is lower, or there is more variability in the values and preferences of patients.

Evidence is graded as high, moderate, low or very low, based on how likely further research is to change our confidence in the estimate of effect (Appendix 2, available at www.cmaj.ca/www.cmaj.ca/lookup/suppl/ doi:10.1503/cmaj.151421/-/DC1).
Embase up to May 2012. An updated search from May 2012 to Mar. 31, 2015, was performed using the same databases and search terms as the Cochrane review. Studies identified in the Cochrane review that also met the review criteria from the Evidence Review and Synthesis Centre were retrieved, and the data were extracted and included in the GRADE decision tables. For harms of screening, a new search was conducted for randomized controlled trials (RCTs) and observational studies to ensure that all literature related to the harms ranked as critical were identified; CENTRAL, MEDLINE, PREMEDLINE, and Embase were searched from 2000 to Mar. 31, 2015. The complete systematic review is available at www.canadiantaskforce.ca.

An updated prepublicaton search was conducted in MEDLINE, Embase and CENTRAL from Mar. 31, 2015 to Jan. 20, 2016, which focused only on RCTs that addressed our critical outcomes.

The recommendations were revised and approved by the entire task force and underwent external review by academic and clinical experts in the area.

The task force uses a rigorous usability testing process to develop knowledge translation tools targeting various end-user groups (e.g., clinicians and patients) to accompany its guidelines. All tools are informed by feedback from clinicians (for clinician and patient tools) and patients (for patient tools) obtained through interviews, focus groups or surveys.

More information about the task force's methods can be found elsewhere ${ }^{11}$ and at the task force website (http://canadiantaskforce.ca/ methods/methods-manual/).

\section{Recommendations}

We recommend screening for lung cancer among adults 55 to 74 years of age with at least a 30 pack-year smoking history, who smoke or quit smoking less than 15 years ago, with low-dose computed tomography (CT) every year up to three consecutive years. Screening should only be done in health care settings with access to expertise in early diagnosis and treatment of lung cancer. (Weak recommendation, low-quality evidence.)

We recommend not screening all other adults, regardless of age, smoking history or other risk factors, for lung cancer with lowdose CT. (Strong recommendation, very lowquality evidence.)

We recommend that chest radiography, with or without sputum cytology, not be used to screen for lung cancer. (Strong recommendation, low-quality evidence.) 
A summary of the recommendations is shown in Box 2, and a clinical summary is provided in Appendix 2 (available at www.cmaj.ca/ lookup/suppl/doi:10.1503/cmaj.151421/-/DC1). The task force based the recommendations on the overall balance between the possible benefits and harms of screening for lung cancer, weighing the potential benefits of early disease detection against the harms of overdiagnosis and invasive follow-up testing.

These recommendations apply to adults aged 18 years and older who are not suspected of having lung cancer. These recommendations do not apply to individuals with previous lung cancer, or signs or symptoms of lung cancer.

\section{Benefits of screening}

The systematic review performed for the task force included 33 studies on lung cancer screening; 13 RCTs studied the benefits of screening. ${ }^{12}$ Seven low-quality studies evaluated screening with chest radiography (with or without sputum cytology), compared with no screening or less intensive screening (e.g., screening with chest radiography at longer intervals or advice to have a chest radiograph) and found small benefits in terms of early disease detection. Screening with chest radiography detected more early-stage and fewer late-stage lung cancers compared with groups receiving usual care. However, such screening did not reduce lung cancer specific mortality (risk ratio [RR] 0.99, 95\% confidence interval [CI] 0.92-1.07]) or all-cause mortality (RR 0.98, 95\% CI 0.96-1.00) when compared with usual care. ${ }^{12}$

Three low-quality trials compared annual screening with low-dose CT to no screening or usual care and found no difference in lung cancer-specific mortality (RR $1.30,95 \%$ CI $0.81-$ 2.11 ) or all-cause mortality (RR $1.38,95 \% \mathrm{CI}$ 0.86-2.22) after five years or less of followup. ${ }^{12}$ A recent update of the literature search found one randomized study ${ }^{15}$ presenting interim results on early detection of lung cancer with low-dose multislice CT (compared with no screening) in Germany after five years of follow-up. The addition of these new results to the all-cause mortality analysis did not significantly alter the results (RR 1.20, 95\% CI 0.83-1.73).

Two studies were found that compared lowdose CT to chest radiography. Although screening with chest radiography is not considered standard care in Canada, these studies were included in the evidence review because prior studies had shown no differences in mortality outcomes between chest radiography and usual care. One of the studies included mortality outcomes (National Lung Screening Trial). ${ }^{6}$ The
National Lung Screening Trial (a high-quality $\mathrm{RCT}$ ) reported a $15 \%$ reduction in lung cancer mortality (RR $0.85,95 \%$ CI $0.75-0.96$ ) and a $6 \%$ reduction in all-cause mortality (RR 0.94 , 95\% CI 0.88-1.00) associated with screening with low-dose CT compared with chest radiography after 6.5 years of follow-up. ${ }^{16}$ This means that screening 1000 people with lowdose CT three times at one-year intervals prevents three deaths from lung cancer compared with screening with chest radiography (number needed to screen $=322$ ). Screening with lowdose CT reduced the absolute risk of lung cancer mortality by $0.31 \%$ and of all-cause mortality by $0.46 \% .{ }^{12}$ Low-dose CT also detected significantly more cases of early-stage lung cancer ( 8 more per 1000 people screened) and significantly fewer cases of late-stage lung cancer (4 fewer per 1000 people screened) compared with chest radiography. Results from the second study ${ }^{17}$ comparing low-dose CT and chest radiography were not pooled with results of the National Lung Screening Trial in the systematic review because of an incompatible follow-up period $(\leq 12 \mathrm{mo})$, the small number of reported events (lung cancers) and no reporting of mortality outcomes. ${ }^{12}$

Evidence from four studies showed no significant differences in rates of smoking cessation between the screened (low-dose CT or chest radiography) groups and the control groups. ${ }^{12}$ The risk of bias for these studies was unclear because of the self-reported nature of this outcome.

\section{Harms of screening}

The harms of screening and invasive follow-up tests were informed by 31 studies, many with observational designs. ${ }^{12}$ The main harms

\section{Box 2: Summary of recommendations for clinicians and policy-} makers

These recommendations apply to adults 18 years of age and older who are not suspected of having lung cancer. These recommendations do not apply to adults with previous lung cancer, or signs or symptoms of lung cancer.

We recommend screening for lung cancer among adults aged 55 to 74 years with at least a 30 pack-year smoking history, who smoke or quit smoking less than 15 years ago, with low-dose computed tomography (CT) every year up to three consecutive years. Screening should only be done in health care settings with access to expertise in early diagnosis and treatment of lung cancer. (Weak recommendation, low-quality evidence.)

We recommend not screening all other adults, regardless of age, smoking history or other risk factors, for lung cancer with low-dose CT. (Strong recommendation, very low-quality evidence.)

We recommend that chest radiography, with or without sputum cytology, not be used to screen for lung cancer. (Strong recommendation, low-quality evidence.) 
included false-positive results, death or major complications from invasive follow-up testing and overdiagnosis.

Data from the National Lung Screening Trial suggest that of every 1000 people screened three times with low-dose CT at one-year intervals, 391 would have at least one positive result, 40 would have lung cancer and 351 would have a false-positive result. ${ }^{6,12}$ As such, follow-up tests, including minor invasive procedures (e.g., bronchoscopy, needle biopsy) or major invasive procedures (e.g., thoracotomy, thoracoscopy), are needed to determine whether a positive low-dose CT result is due to lung cancer. Although uncommon, there is a risk of major complications or death with these procedures. Based on data from the National Lung Screening Trial, 3 people per 1000 screened with low-dose CT experience major complications from invasive tests, and less than 1 person per 1000 screened die after an invasive test (within 60 d). ${ }^{6,12}$ Some of these complications occur in people who receive a false-positive screening result. Data from 17 studies showed that 5 people per 1000 screened with low-dose CT received an unnecessary major invasive procedure for an ultimately benign condition (compared with 3 people per 1000 screened with chest radiography). ${ }^{12}$

Overdiagnosis occurs when people who are asymptomatic undergo screening for lung cancer and a slow-growing cancer that would have never caused them any harm during their lifetime is detected and diagnosed. ${ }^{18}$ Although current estimates of overdiagnosis for lung cancer vary by thresholds used and are based on limited followup, observational studies suggest that $2 \%-16 \%$ of lung cancers detected with chest radiography and $11 \%-26 \%$ of lung cancers detected with lowdose CT represent overdiagnosis. ${ }^{12}$ Overdiagnosis often leads to unnecessary treatment (overtreatment), which can cause harm.

\section{Performance characteristics of screening tools}

The test characteristics of various low-dose CT technologies (e.g., multislice or helical) (but not chest radiography) were examined in the review performed for the task force..$^{12}$ The sensitivity of low-dose CT is high (80\%-100\%), but the specificity varies widely $(28 \%-100 \%)$, likely contributing to the high frequency of false-positive results. Studies reporting on test performance found that a multislice $\mathrm{CT}$, along with computerassisted reading and diagnosis and two independent radiologist readers, showed the highest sensitivity $(94.6 \%)$ and specificity $(98.3 \%) .{ }^{12}$ By comparison, the National Lung Screening Trial trial used low-dose helical CT without computerassisted reading and diagnosis and one radiolo- gist reader, and reported a sensitivity of $94.4 \%$ and specificity of $72.6 \% .6,12$ The cut points for a positive low-dose CT result varied across studies (ranging from $>3$ to $>10 \mathrm{~mm}$ ). At present, there is no agreement on which cut point will optimize the balance between a reduction in mortality and minimizing harm.

\section{Rationale}

The findings of the National Lung Screening Trial ${ }^{6}$ suggest that screening with low-dose CT may reduce lung cancer mortality in patients at high risk. However, this benefit was shown in only one $\mathrm{RCT}$, and there are possible harms related to screening and invasive follow-up testing. In addition, although there is no reason to suspect that screening with chest radiography would increase the risk of death from lung cancer, the National Lung Screening Trial compared screening with low-dose CT versus chest radiography rather than comparing low-dose CT with no screening (indirect evidence). For these reasons, the task force has made a weak recommendation to offer screening with low-dose $\mathrm{CT}$ for men and women at high risk of lung cancer (adults 55-74 years of age with at least a 30 pack-year smoking history who smoke or used to smoke). The overall body of evidence supporting this recommendation is rated as low quality. Whereas one high-quality study (National Lung Screening Trial) helped inform the recommendation, ${ }^{6}$ additional low-quality studies were used to address the critical outcomes. Following GRADE methods, the rating for the overall body of evidence must be based on the lowest-quality evidence used. Research currently underway may change our level of confidence in the benefit of low-dose CT screening for lung cancer, and we will reassess our recommendations once this new evidence is available.

The recommendation to screen the high-risk population places a higher value on a small benefit for reduced lung cancer mortality and the known poor prognosis of untreated lung cancer, ${ }^{19}$ but a lower value on the risk of adverse effects, overdiagnosis and the lack of data comparing low-dose CT with no screening.

There were no low-dose CT screening studies involving adults at low or moderate risk of lung cancer, or who had other risk factors unrelated to smoking (e.g., exposure to radon), that examined the outcomes the task force identified as critical or important. Therefore, the overall body of evidence was rated as very low quality. Men and women who are not at high risk of lung cancer (i.e., those who smoke with less than 30 packyears of smoking history or those who quit smoking more than 15 years prior) would be expected to have lower absolute benefit of 
screening than the patients at high risk included in the National Lung Screening Trial but would still be susceptible to the potential harms (e.g., false-positive results, consequences from invasive follow-up tests and overdiagnosis) associated with screening. For these reasons, we strongly recommend against screening adults who are not at high risk or who have other risk factors for lung cancer unrelated to smoking.

We have made a strong recommendation against screening with chest radiography based on low-quality evidence because available evidence suggests that there is no benefit of screening with chest radiography on lung cancer-specific or all-cause mortality, but there are established harms (e.g., overdiagnosis, false-positive results and complications from follow-up testing).

\section{Considerations for implementation}

These recommendations apply only to adults who are not suspected of having lung cancer on clinical grounds. In adults with symptoms of lung cancer (e.g., hemoptysis, weight loss, dyspnea), regardless of age or smoking history, clinicians should consider diagnostic testing as clinically indicated.

A weak recommendation means that most eligible adults would want to be screened for lung cancer, but many may appropriately choose not to be screened. Primary care providers should discuss the potential harms and benefits of screening with patients at high risk of developing lung cancer based on age and smoking history. Providers should also consider overall health status when discussing this issue with patients, because reasonable life expectancy and suitability for treatment of lung cancer (if identified) is required to benefit from screening. People who place a higher value on a potential mortality benefit and are less concerned with the harms associated with screening (e.g., high false-positive rate, complications from follow-up testing) will be more likely to choose screening, whereas those more concerned with the harms and small mortality benefit may choose not to be screened.

Because the accuracy of detection and quality of follow-up investigations and management are critical to obtaining more benefit than harm, screening for lung cancer with low-dose CT should only be considered in settings that can deliver comprehensive care similar to or better than that offered in the National Lung Screening Trial $^{6}$ (e.g., centres with qualified radiologists and radiologic technologists, with examinations and diagnostic follow-up guidelines aligned with the study protocol and with expertise in the early diagnosis and management of lung cancer). Incorporation of nodule risk calculators may also decrease risk of overdiagnosis and rates of false-positive results. Implementation of these recommendations in settings without relevant expertise may decrease the benefit:harm ratio, potentially increasing the harms. The task force recognizes that low-dose CT scans and such expertise are not currently accessible in certain regions in Canada (e.g., rural and remote areas), and this is a consideration that policy-makers will need to address.

\section{Patient values and preferences}

Patient preferences on screening for lung cancer were assessed in seven studies included in the systematic review, ${ }^{12}$ as well as de novo data collection conducted for the task force. The systematic review concluded that most participants in the high-risk group (i.e., adults 55-74 years of age who smoke or have quit smoking) had high willingness to participate in screening for lung cancer, motivated by their smoking history, belief that early detection improves health outcomes and a family history of lung cancer. ${ }^{12}$ Potential barriers to participation included inconvenience of screening and negative experiences with health care workers or settings. Patients who were screened for lung cancer with low-dose CT did not feel substantial distress or anxiety. ${ }^{12}$ In the judgment of the task force, the consistent evidence showing that patients at high risk are willing to be screened for lung cancer supports the recommendation for screening in this group.

The task force conducted a series of focus groups and a survey with 15 members of the public (mean age $63 \mathrm{yr}$ [range 36 to $76 \mathrm{yr}$ ], 12 were female and 8 smoked or had quit smoking) to assess patient perceptions of these recommendations for screening for lung cancer. ${ }^{20}$ In general, participants agreed that these recommendations were appropriate, beneficial and feasible, although some had concerns about access to low-dose CT scans and limiting eligibility to adults between 55 and 74 years.

\section{Other considerations}

It is possible that longer or more intensive screening might yield additional benefits, but this is speculative, since there are no RCT data to support such a recommendation. Although ongoing screening (i.e., more than 3 screens) might further reduce mortality, it might also lead to more false-positive results and complicatons from invasive follow-up testing, potentially disrupting the balance between the benefits and harms as reported in the National Lung Screening Trial study. ${ }^{6}$ 
Smoking remains the primary risk factor for lung cancer; therefore, interventions to promote smoking cessation (which have benefits beyond decreasing the risk of lung cancer) should be incorporated into any screening program aimed at reducing lung cancer morbidity and mortality. Smoking cessation resources can be found in the Canadian Best Practices Portal of the Public Health Agency of Canada. ${ }^{21}$

\section{Suggested performance measures}

National-level quality indicators are currently being developed by the Pan-Canadian Lung Cancer Screening Network. ${ }^{22}$ Additional suggested performance measures, although difficult to measure, include rates of discussion of screening for lung cancer using low-dose CT among populations at high risk and the proportion of potentially eligible adults receiving accurate information about the risks and benefits of screening (ideally using an evidence-based decision aid). Reduction in use of chest radiography to screen for lung cancer is a potentially important implementation measure. Harms of screening and follow-up tests in different settings should also be continually assessed. Incidence and mortality data related to lung cancer should continue to be monitored at the provincial, territorial and national levels.

\section{Economic implications}

Data from a microsimulation model (the Cancer Risk Management Model) were used to assess the costs and consequences of screening for lung cancer in Canada as recommended in the current guideline. ${ }^{23}$ This model is based on the results of the National Lung Screening Trial but compares the costs and consequences of low-dose CT screening with no screening. We assessed the model according to task force processes, and it was deemed appropriate for use in this context. ${ }^{24}$

Based on a scenario aligned with the current guideline, and assuming that $100 \%$ of patients meeting the eligibility criteria of the National Lung Screening Trial participated in screening (with 95\% participating in rescreening as recommended), the incremental system cost would be $\$ 2.3$ billion between 2014 and 2034. Although indirect costs were not incorporated in the model, the incremental cost-effectiveness ratio, compared with no screening, would be $\$ 74000$ per quality-adjusted life-year, ${ }^{23}$ suggesting that (if done in centres with appropriate expertise) low-dose CT screening could represent reasonable value for money.

Several factors affect these estimates, including assumptions about rates of screening participation and rescreening compliance. However, screening remained economically attractive using conventional thresholds when rescreening adherence rates were varied within plausible ranges. ${ }^{25}$ In addition, low-dose CT screening appeared to be more economically attractive when combined with a smoking cessation program. ${ }^{23}$

\section{Other guidelines}

These new recommendations generally align with other Canadian and international guidelines on screening for lung cancer (Table 1), ${ }^{4,5,26-32}$ However, the task force is taking a more conservative approach in recommending three annual scans rather than continuous annual or biennial scans.

\section{Gaps in knowledge}

There is limited evidence for the optimal duration (e.g., ongoing screening after the initial 3 annual scans) and interval (e.g., annual, biennial) for lung cancer screening. In addition, the cut point for a positive scan result varied across studies. Further research is needed to determine optimal management of lung nodules and diagnostic practices. Until such evidence is available, the protocol used in the National Lung Screening Trial should be used. ${ }^{6}$

Further research is needed to determine whether risk assessment tools could be incorporated into the clinical algorithm, and what impact this would have on patient-important outcomes. Whether adults with other risk factors for lung cancer (e.g., exposure to radon, second-hand smoke and other toxic substances; occupational exposures; family history or prior radiation to the chest) or with less extensive smoking histories may also benefit from screening with low-dose CT is unknown. Better data on how to identify those who will not benefit from screening because of competing morbidities should be a high priority for future research.

\section{Conclusion}

The task force recommends screening for lung cancer with three consecutive annual low-dose CT scans among adults 55 to 74 years of age, with at least a 30 pack-year history of smoking, who smoke or who quit smoking within the previous 15 years. Among all other adults, regardless of age, smoking history or other risk factors, the task force recommends not to routinely screen for lung cancer with low-dose CT. The task force recommends not screening for lung cancer using chest radiography (with or without sputum cytology). 
Table 1: Summary of recommendations for screening for lung cancer from Canada and elsewhere

Organization Recommendation

Canadian Task Force on

Preventive Health Care (current)

Canadian Task Force on

Preventive Health Care (2003) $)^{5}$

US Preventive Services Task Force $(2013)^{4}$

Cancer Care Ontario (2013) ${ }^{26}$

American Cancer Society $(2013)^{27}$

American College of Chest Physicians (2013) ${ }^{28}$

American Lung Association (2012, updated 2015) ${ }^{29}$

American Association for Thoracic Surgery (2012) ${ }^{30}$ Network (2015) ${ }^{31}$

American Academy of Family Physicians (2013) ${ }^{32}$
Screen asymptomatic adults aged 55 to 74 years with at least a 30 pack-year smoking history who smoke or quit smoking less than 15 years ago for lung cancer with low-dose CT every year for 3 consecutive years

Do not screen asymptomatic adults for lung cancer with chest radiography; insufficient evidence for using low-dose CT as a screening test for asymptomatic adults

Screen asymptomatic adults aged 55 to 80 years with a minimum 30 pack-year smoking history who smoke or quit smoking within the last 15 years for lung cancer with low-dose CT annually

Screen high-risk populations (i.e., adults 55-74 yr of age with a minimum smoking history of 30 pack-years or more who currently smoke or quit smoking within the past $15 \mathrm{yr}$ and are free of disease at the time of screening) for lung cancer with low-dose CT for 3 years followed by biennial screening

Screen adults aged 55 to 74 years with a 30 pack-year or more smoking history who smoke or quit smoking within the previous 15 years and are in relatively good health for lung cancer with low-dose CT annually

Screen adults aged 55 to 74 years with a 30 pack-year or more smoking history who smoke or quit smoking within the previous 15 years for lung cancer with low-dose CT annually

Screen adults aged 55 to 74 years with a smoking history of at least 30 pack-years and no history of lung cancer who smoke or quit smoking with the last 15 years for lung cancer using low-dose CT annually

Screen adults aged 55 to 79 years with a 30 pack-year or more smoking history; adults with a previous diagnosis of lung cancer who have completed 4 years of surveillance without recurrence, and who can tolerate lung cancer treatment following screening to detect second primary lung cancer until the age of 79 years; and adults aged 50 to 79 years with a 20 pack-year smoking history and an additional comorbidity that produces a cumulative risk of lung cancer of $5 \%$ or more in 5 years for lung cancer with low-dose CT annually

Screen adults aged 55 to 74 years with a 30 pack-year or more smoking history who quit smoking less than 15 years ago, and adults aged 50 years or older with a 20 pack-year or more smoking history and 1 additional risk factor (other than exposure to second-hand smoke) for lung cancer with low-dose CT annually

Insufficient evidence to recommend for or against screening for lung cancer with low-dose CT

Note: $\mathrm{CT}=$ computed tomography.

\section{References}

1. Canadian Cancer Society's Advisory Committee on Cancer Statistics. Canadian Cancer Statistics 2015. Toronto: Canadian Cancer Society; 2015.

2. Canadian Tobacco Use Monitoring Survey (CTUMS) 2012 Ottawa: Health Canada; 2012. Available: www.hc-sc.gc.ca/hc-ps/ tobac-tabac/research-recherche/stat/ctums-esutc_2012-eng.php (accessed 2014 Jan. 22)

3. Non-small cell lung cancer survival rates by stage. Atlanta: American Cancer Society; 2015. Available: www.cancer.org/cancer/ lungcancer-non-smallcell/detailedguide/non-small-cell-lung-cancer -survival-rates (accessed 2015 May 20).

4. US Preventive Services Task Force. Lung cancer screening: recommendation statement. Ann Intern Med 2004;140:738-9.

5. Palda VA, Van Spall HGC. Screening for lung cancer: updated recommendations from the Canadian Task Force on Preventive Health Care. Calgary: Canadian Task Force on Preventive Health Care; 2003. Available: canadiantaskforce.ca/files/ guidelines/2003-lung-cancer-updated-recommendations-en.pdf (accessed 2016 Jan. 29)

6. Aberle DR, Adams AM, Berg CD, National Lung Screening Trial Research Team; et al. Reduced lung-cancer mortality with low-dose computed tomographic screening. $N$ Engl J Med 2011;365:395-409

7. Lopes Pegna A, Picozzi G, Mascalchi M, et al. Design, recruitment and baseline results of the ITALUNG trial for lung cancer screening with low-dose CT. Lung Cancer 2009;64:34-40.

8. van Iersel CA, de Koning HJ, Draisma G, et al. Risk-based selection from the general population in a screening trial: selection criteria, recruitment and power for the Dutch-Belgian randomised lung cancer multi-slice CT screening trial (NELSON). Int J Cancer 2007;120:868-74.

9. Saghir Z, Dirksen A, Ashraf H, et al. CT screening for lung cancer brings forward early disease. The randomised Danish Lung Cancer Screening Trial: status after five annual screening rounds with low-dose CT. Thorax 2012;67:296-301.

10. Becker N, Motsch E, Gross ML, et al. Randomized study on early detection of lung cancer with MSCT in Germany: study design and results of the first screening round. J Cancer Res Clin Oncol 2012;138:1475-86.

11. Canadian Task Force on Preventive Health Care procedure manual. Calgary: Canadian Task Force on Preventive Health Care; 2014. Available: canadiantaskforce.ca/files/procedural-manualen.pdf (accessed 2016 Jan. 29)

12. Peirson L, Ali MU, Warren R, et al. Screening for lung cancer: systematic review and meta-analyses. Hamilton (ON): McMaster Evidence Review and Synthesis Centre; 2015. Available: canadian taskforce.ca/ctfphc-guidelines/2015-lung-cancer/systematic-review (accessed 2016 Feb. 25).

13. Schünemann $H$, Brozek J, Guyatt $G$, et al, editors. GRADE handbook. The GRADE Working Group; 2009. Available: gdt. guidelinedevelopment.org/central_prod/_design/client/hand book/handbook.html\#h.17zdc2r2pkyf (accessed 2016 Jan. 29).

14. Manser R, Lethaby A, Irving LB, et al. Screening for lung cancer. Cochrane Database Syst Rev 2013;(6):CD001991.

15. Becker N, Motsch E, Gross ML, et al. Randomized study on early detection of lung cancer with MSCT in Germany: results of the first 3 years of follow-up after randomization. $J$ Thorac Oncol 2015;10:890-6. 
16. Black WC, Keeler EB, Soneji SS, et al. Cost-effectiveness of CT screening in the National Lung Screening Trial. N Engl J Med 2015;372:388.

17. Gohagan J, Marcus P, Fagerstrom R, et al. Baseline findings of a randomized feasibility trial of lung cancer screening with spiral CT scan vs chest radiograph: the Lung Screening Study of the National Cancer Institute. Chest 2004;126:114-21.

18. Marcus PM, Prorok PC, Miller AB, et al. Conceptualizing overdiagnosis in cancer screening. J Natl Cancer Inst 2015;107:djv014

19. Wao H, Mhaskar R, Kumar A, et al. Survival of patients with non-small cell lung cancer without treatment: a systematic review and meta-analysis. Syst Rev 2013;2:10.

20. Bashir N, Mascarenhas A, Moore J, et al. Patient preferences for Lung Cancer Screening Guideline recommendations. Toronto: La Ka Shing Institute, St. Michael's Hospital; 2015 Available: canadiantaskforce.ca/files/ctfphcpatientpreference slungcancerreport 150706final.pdf (accessed 2016 Feb. 25).

21. Canadian Best Practices Portal: interventions. Ottawa: Public Health Agency of Canada; 2015. Available: cbpp-pcpe.phac -aspc.gc.ca/interventions/ (accessed 2015 Oct. 9).

22. Lung cancer screening framework can help maximize benefits, reduce risks. Toronto: Canadian Partnership Against Cancer; 2015. Available: www.partnershipagainstcancer.ca/lung-cancer -screening-framework-can-help-maximize-benefits-reduce-risks/ (accessed 2015 Oct. 9).

23. Goffin JR, Flanagan WM, Miller AB, et al. Cost-effectiveness of lung cancer screening in Canada. JAMA Oncol 2015;1:807-13.

24. Sadatsafavi M, Zafari Z, Mitton C, et al. Evaluating the Cancer Risk Management Model (CRMM) - Lung Cancer Module. Vancouver: Centre for Clinical Epidemiology and Evaluation, Vancouver Coastal Health Research Institute; 2015. Available: canadiantaskforce.ca/files/ubc-eval-crmm-lc-final.pdf (accessed 2016 Feb. 25).

25. Clement FM, Harris A, Li JJ, et al. Using effectiveness and costeffectiveness to make drug coverage decisions: a comparison of Britain, Australia, and Canada. JAMA 2009;302:1437-43.

26. Roberts H, Walker-Dilks C, Sivjee K, et al. Screening high-risk populations for lung cancer. Toronto: Cancer Care Ontario; 2013

27. Lung cancer. Atlanta (GA): American Cancer Society; 2014. Available: www.cancer.org/cancer/lungcancer/index (accessed 2014 Apr. 2).

28. Alberts WM; American College of Chest Physicians. Introduction: diagnosis and management of lung cancer: ACCP evidence-based clinical practice guidelines. Chest 2007;132(3 Suppl):20S-22S.

29. Providing guidance on lung cancer screening to patients and physicians. Washington: American Lung Association; 2012. Available: www.lung.org/assets/documents/lung-cancer/lung-cancer -screening-report.pdf (accessed 2013 Sept. 13).

30. Jaklitsch MT, Jacobson FL, Austin JH, et al. The American Association for Thoracic Surgery guidelines for lung cancer screening using low-dose computed tomography scans for lung cancer survivors and other high-risk groups. J Thorac Cardiovasc Surg 2012;144:33-8.

31. NCCN Guidelines for Patients. Lung cancer screening. Fort Washington (PA): National Comprehensive Cancer Network (NCCN); 2014. Available: www.nccn.org/patients/guidelines/ lung_screening/index.html (accessed 2013 Sept. 22).

32. Clinical preventive service recommendation: lung cancer Leawood (KS): American Academy of Family Physicians; 2013. Available: www.aafp.org/patient-care/clinical-recommendations/ all/lung-cancer.html (accessed 2015 May 22).

Guideline writing group: Gabriela Lewin, Kate Morissette, James Dickinson, Neil Bell, Maria Bacchus, Harminder Singh, Marcello Tonelli, Alejandra Jaramillo Garcia

Affiliations: Department of Family Medicine (Lewin), University of Ottawa; Public Health Agency of Canada
(Morissette, Jaramillo Garcia), Ottawa, Ont; Department of Family Medicine (Bell), University of Alberta, Edmonton, Alta.; Departments of Internal Medicine and Community Health Sciences (Singh), University of Manitoba, Winnipeg, Man.; Departments of Family Medicine and Community Health Sciences (Dickinson), Department of Medicine (Bacchus), and Office of the Associate Dean (Research) (Tonelli), University of Calgary, Calgary, Alta.

Contributors: Gabriela Lewin, James Dickinson, Neil Bell, Maria Bacchus, Harminder Singh, Alejandra Jaramillo Garcia and Marcello Tonelli contributed substantially to the study concept and design, and interpretation of the evidence, and revised the draft. Kate Morissette contributed to the analysis and interpretation of the evidence and drafted the manuscript. All of the authors gave final approval of the version to be published and agreed to act as guarantors of the work.

Funding: Funding for the Canadian Task Force on Preventive Health Care is provided by the Public Health Agency of Canada. The Cancer Risk Management Model has been made possible through a financial contribution from Health Canada, through the Canadian Partnership Against Cancer. The views of the funding bodies have not influenced the content of the guideline; competing interests have been recorded and addressed. The views expressed in this article are those of the authors and do not necessarily represent those of the Public Health Agency of Canada.

Acknowledgements: The authors acknowledge members of the Evidence Review and Synthesis Centre research team located at McMaster University (Leslea Peirson, Muhammad Usman Ali, Donna Fitzpatrick-Lewis, Parminder Raina, Diana Scherifali and Meghan Kenny) who conducted the systematic review upon which these recommendations were based; the staff at the Prevention Guidelines Division of the Public Health Agency of Canada; the team at the Centre for Clinical Epidemiology and Evaluation, University of British Columbia, who conducted the evaluation of the Cancer Risk Management Model - Lung Cancer Module (Mohsen Sadat Safavi, Zafar Zafari, Craig Mitton and Sirling Bryan); and the reviewers whose comments helped the authors to improve this manuscript (Melissa Brouwers, McMaster University and the Escarpment Cancer Research Institute; Heather Bryant, Canadian Partnership Against Cancer and University of Calgary; Jennifer Crosswell, Agency for Healthcare Research and Quality; Conrad B. Falkson, Queen's University and Cancer Centre of Southeastern Ontario; Tunji Fatoye, University of Manitoba and CancerCare Manitoba; John R. Goffin, McMaster University; Christopher A. Hergott, University of Calgary; Michael R. Johnston, Beatrice Hunter Cancer Research Institute, Terry Fox Research Institute and Dalhousie University; Alan Kaplan, The College of Family Physicians Canada; John C. Kirk, McGill University; Mark Kristjanson, University of Manitoba and CancerCare Manitoba; Stephen Lam, University of British Columbia and BC Cancer Agency; Suzanne Levitz, The College of Family Physicians Canada; Paul MacEachern, University of Calgary; Robert Nuttall, Canadian Cancer Society; and Gilles Plourde, Health Canada). 